\title{
MANUFACTURING AND MECHANICAL BEHAVIOR OF THE GRAPHITE NANOPLATELETS / AI MATRIX COMPOSITES
}

\author{
A.I. Selmy, F. Shehata, E. Gewfiel \\ Mechanical Design and Production Engineering Department, \\ Faculty of Engineering, Zagazig University, Zagazig, Egypt. \\ E-mail:Drselmy@hotmail.com
}

\begin{abstract}
One of the main objectives of this work is to manufacture the reinforcing graphite nanoplatelets (CNPLs)] materials in the laboratory from the natural graphite. Also, aluminum matrix composite reinforced with 1, 2, 3, 4 and 5 wt.\% graphite nanoplatelets has been fabricated by cold pressing, followed by hot extrusion techniques. Scanning electron microscopy (SEM) has been used to examine the dispersion of CNPLs and aluminum, and to analyze the composites structure. The microstructure characteristics and the distribution of CNPLs in the aluminum matrix were investigated. The mechanical properties of the composites were recorded at room temperature. Experimental results showed that CNPLs were distributed homogeneously in the (CNPLs/Al) composites. The CNPLs content affects significantly on the mechanical properties of (CNPLs/Al) composites. Meanwhile, the $2.0 \mathrm{wt} \%$ CNPLs /Al composite is found to exhibit the highest hardness, yield and ultimate tensile strengths. Also, it is noticed that the elongation percentages remain nearly constant. The extraordinary mechanical properties (yield and ultimate tensile strengths) of CNPLs may be the reason for this increments, in additional to the bridging and pulling-out role of CNPLs in the aluminum matrix composites. The tightly bonded interface between the matrix and CNPLs can also effectively transfer the load to the graphite nanoplatelets. Further enhanced strength has been achieved by reinforcing the matrix with dispersed nanoplatelets in such composites.
\end{abstract}

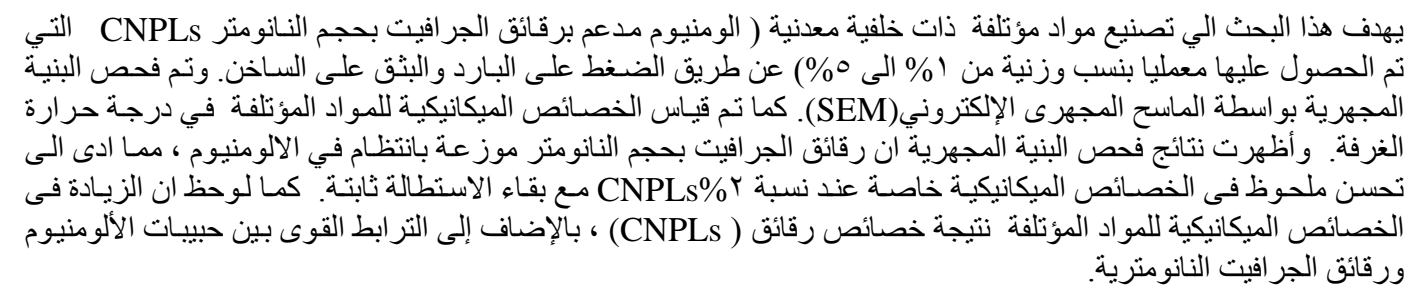

Keywords: Composite; graphite nanoplatelets; cold press; hot extrusion; mechanical properties.

\section{INTRODUCTION}

Aluminum alloys have a great diversity of industrial applications because of their low density and good workability, but the use of these alloys is limited due to their relatively low yield strength. Recently, the interest to increase aluminum strength for applications has motivated the study of aluminum matrix composites. The main reasons to produce aluminum matrix composites are to increase the strength, stiffness and wear resistance of aluminum. Aluminum can be strengthened by dispersing hard particles like carbides, oxides or graphite's into the aluminum matrix [1,2].

It can also be fabricated in the solid state through Powder metallurgy (PM) techniques, which are widely used due to their great versatility and low cost of production. The process of fabrication consists in mixing the hardening particles with the metallic powders followed by consolidation and sintering. Even though graphite acts as an excellent lubricating agent under conditions of friction, graphite dispersion in aluminum has not been deeply investigated. Carbon nanoplatelets probably offer a kind of nanosize reinforcement that is lightweight, 
has immense aspect ratio, and has remarkable mechanical, electrical and thermal properties [3]. A very limited research has been done in the field of graphite nanoplatelets reinforced metal matrix composites due to the fact that uniform dispersion of graphite nanoplatelets in metal matrix is quite difficult. The interfacial reaction between graphite nanoplatelets and metal matrix may be rather serious resulting in the deterioration of composite properties, and the suitable fabrication technique also is important.

Graphene, consisting of a single layer of carbon in a two dimensional (2D) lattice, has been emerging as a fascinating material with many unique physical, chemical and mechanical properties [4,5]. Graphite is a one atom thick layered. In general, graphene sheets can be prepared by three techniques: (i) micromechanical cleavage, producing graphene sheets in very limited quantities, (ii) epitaxial growth of graphene films, and (iii) chemical processing, involving graphite oxidation, exfoliation and reduction. Graphite is a 3D network of graphene and is inexpensive (from either natural or synthetic sources).

Graphite can be intercalated by exposing it to appropriate atoms or molecules, known as the intercalating agent, which enter between the carbon layers of the graphite. The resulting material, known as a graphite intercalation compound (GIC), is composed of carbon layers and intercalated layers stacked on top of one another in a periodic fashion. The number of carbon layers between each pair of intercalated layers is called the stage [6]. Rapid heating of intercalated graphite flakes to a sufficiently high temperature causes exfoliation, a sudden increase in the dimension perpendicular to the carbon layers of the GIC. This forms vermicular graphite, also known as expanded graphite. The expanded graphite here was loose and vermicular or wormlike. The exfoliated graphite flakes sonicated in an alcohol solution and obtained graphite powder are shown in Fig.1. As metal powder size is much larger than that of graphite nanoplatelets, it is difficult to achieve homogeneous distribution of graphite nanoplatelets in the composites.

To solve this problem, Noguchi et al. [8] reported a nano-scale dispersion method in carbon nanotube/Al (CNT/Al) composites by introducing into anelastomer precursor. Cha et al. [9] found a molecular level mixing method in carbon nanotube/Copper $(\mathrm{CNT} / \mathrm{Cu})$ composites by means of a salt containing $\mathrm{Cu}$ ions. Furthermore, Hu et al. [10] showed an in situ reduction approach in carbon nanotube/sliver(CNT/Ag) nano- particle composites materials. However, it is believed that under the proper processing condition the CNPLs can be dispersed in the aluminum matrix and keep their good structure and the properties of the aluminum matrix will be improved.

In the present work, CNPLs had been manufactured in the laboratory from natural graphite. A novel processing approach has been undertaken to fabricate a Al-CNPLs nano-structural composite by cold pressing, followed by hot extrusion techniques. The investigations of the microstructure and the mechanical characterization of the composites are reported.

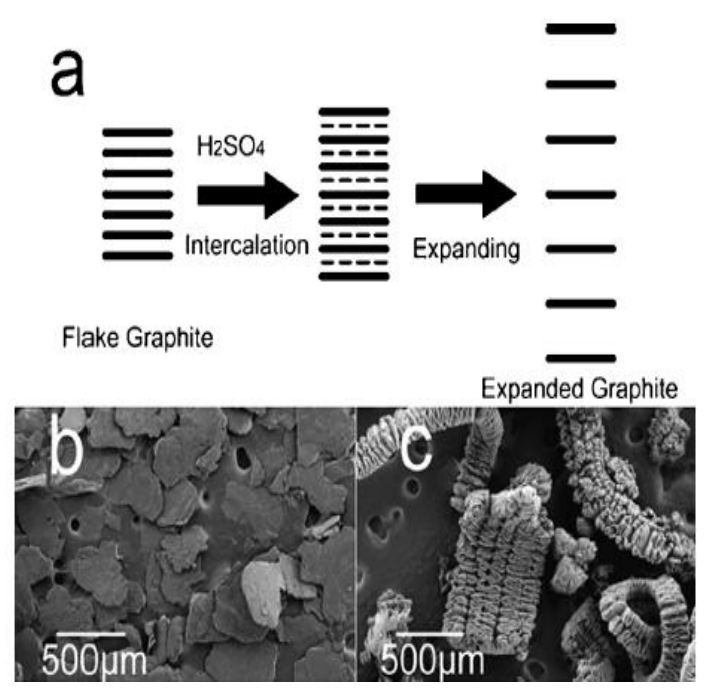

Fig. 1 (a) Mechanism of expanding process, (b) the SEM micrograph of flaky graphite and (c) the SEM micrograph of expanded graphite[7].

\section{EXPERIMENTAL WORK}

\subsection{Manufacturing of CNPLs}

The graphite used for preparing the expanded graphite was natural flake graphite with an average size of $500 \mu \mathrm{m}$. Expanded graphite was prepared according to literature $[11,12]$. A mixture of concentrated sulfuric acid and nitric acid (4:1, v/v) was mixed with graphite flake at room temperature. The reaction mixture was stirred continuously for 16 h. The acid-treated natural graphite was washed with water until neutralized and was then dried at 100 0C to remove any remaining water. The dried particles were heat-treated at $10500 \mathrm{C}$ for $15 \mathrm{~s}$ to obtain expanded graphite particles. The above expanded graphite was immersed in $70 \%$ alcohol solution in an ultrasonic bath. The dispersion was filtered and dried after $8 \mathrm{~h}$ of sonication. The graphite powder, were called graphite nanosheets were kept for testing and for further use. Fig.2. summaries the manufacturing process of CNPLs. 


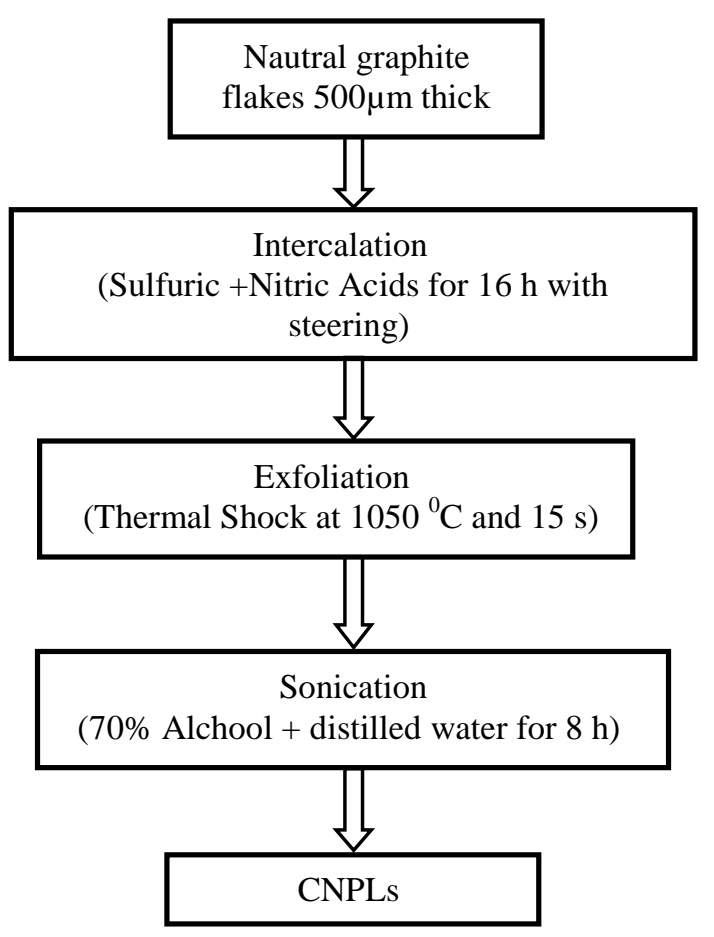

Fig.2 Manufacturing of graphite nanoplatelets (CNPLs) from natural graphite

\subsection{Manufacturing of CNPLs/Al composites}

Aluminum powder (Aluminum Powder Company, Anglesey, UK, 99.4\% pure, $150 \mu \mathrm{m}$ size) was used as the matrix, it has the chemical composition of $0.2 \% \mathrm{Si}, 0.15 \% \mathrm{Fe}, 0.1 \% \mathrm{Cu}, 0.1 \% \mathrm{Mg}, 0.05 \% \mathrm{Mn}$ and the rest is Al. Five CNPLs wt $\%(1,2,3,4$ and $5)$ were added to the aluminum powder. The raw CNPLs were refluxed in nitric acid (68 wt.\%) for 10 $\mathrm{h}$ at $1200 \mathrm{C}$, and then carbon CNPLs were washed several times with distilled water until the washings show no acidity; finally they were added to the dimethylformamide (DMF) in order to retain uniform distribution. Subsequently, Al powders were introduced into the CNPLs-DMF solution and the mixed powders were dispersed with mechanical stirring for $30 \mathrm{~min}$. Finally, the mixed powders were dried at $1200 \mathrm{C}$. The mixed powders were densified by cold pressing at $400 \mathrm{MPa}$ for $30 \mathrm{~min}$. After cold pressing, the composite billets were finally extruded into rods (dia. $=10 \mathrm{~mm}$ ) at $5000 \mathrm{C}$ with an extrusion ratio of $16: 1$. In order to compare with the composites, Al matrix material also was fabricated under the same processing conditions.

2.3 Characterization of CNPLs/Al composites

All the tested samples were machined from the middle portion of the as-fabricated materials. The relative density of composite is a ratio of the measured density to theoretical density, multiplied by 100 . The density of the composites was measured by the Archimedes method, using water immersion. The micro-hardness indentations were made by means of a Vickers diamond indenter operating at a load of $50 \mathrm{~g}$ and dwell time of $12 \mathrm{~s}$. The tensile tests were performed with a cross head speed of 0.5 $\mathrm{mm} / \mathrm{min}$ to obtain the mechanical properties of the composites with different contents of graphite nanoplatelets. For tension tests, shaped samples were used in according to ASTM standard (E8, 2006). Each tensile test data was obtained from the average of five specimens. To investigate the distribution of graphite nanoplatelets in the composite and the interfacial property between the CNPLs and the Al matrix, scanning electron microscopy (SEM) was used to examine the fracture surfaces of the tensile specimens.

\section{RESULTS AND DISCUSSION S}

\subsection{Characterization of CNPLs and Al powders}

Figure 3(a) shows the starting aluminum powder micron-sized used as a matrix. Exfoliated graphite before and after ultrasonic irradiation is shown in Figs. 3(b) and 3(c). The SEM image of graphite sheets prepared upon $8 \mathrm{~h}$ ultrasonic irradiation, clearly, exfoliated graphite have been completely changed into sheets of CNPLs 5-20 $\mu \mathrm{m}$ in diameter and $30-60 \mathrm{~nm}$ in thickness, named graphite nanosheets [11].
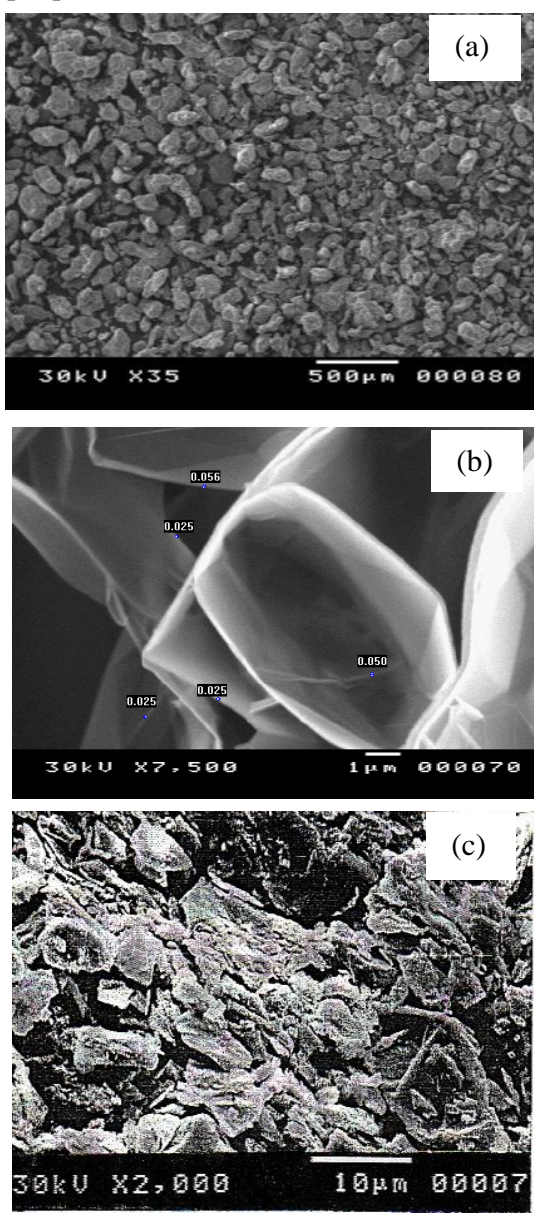

Fig. 3 SEM micrographs of the received powders (a) Pure Al; (b) CNPLs before sonication; (c) CNPLs after sonication 
Figure (4) illustrates the SEM images of homogeneously blended powders of graphite nanoplatelets (CNPLs) and Al powders. It is noticed that the graphite nanoplatelets (CNPLs) are distributed on the surfaces of the Al powders; no agglomeration of graphite nanoplatelets in the powder mixture.
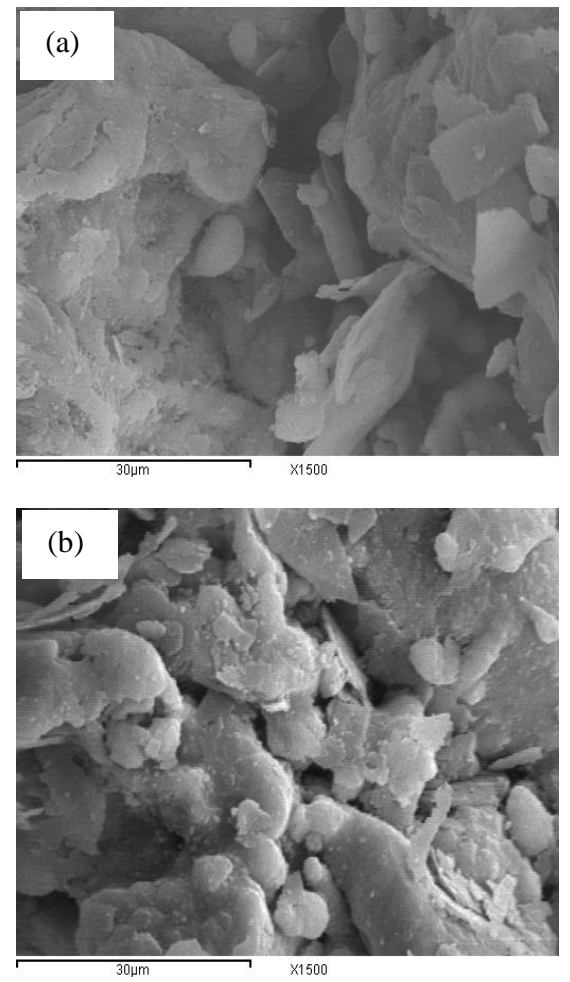

Fig. 4. SEM micrographs of the mixed powders with (a) 2.0 wt.\% CNPLs; (b) 4.0 wt.\% CNPLs.

\subsection{Morphology of CNPLs/Al composites}

Figure 5(a\&b) shows the SEM micrographs of Al2 wt.\% CNPLs and Al-4 wt.\% CNPLs composite after extrusion. The SEM micrograph examination carried on the surface perpendicular to the extrusion direction. It was found that the weight percent of graphite within the powder mixture did not have any considerable effect on its compaction behavior. The best pressure for cold compaction of $\mathrm{Al}$ powder blends containing $0,1,2,3,4$ and 5 wt. $\%$ of graphite particles was $400 \mathrm{MPa}$ at which the porosity of samples was measured to vary within the range of 39 vol.\%.

The dark regions represent the pores or voids, which were left behind by evacuation of graphite particles from surfaces during the polishing process. It can be seen that the graphite particles have been distributed uniformly within the matrix, due to a better dispersion of CNPLs in dimethylformamide (DMF) solution.
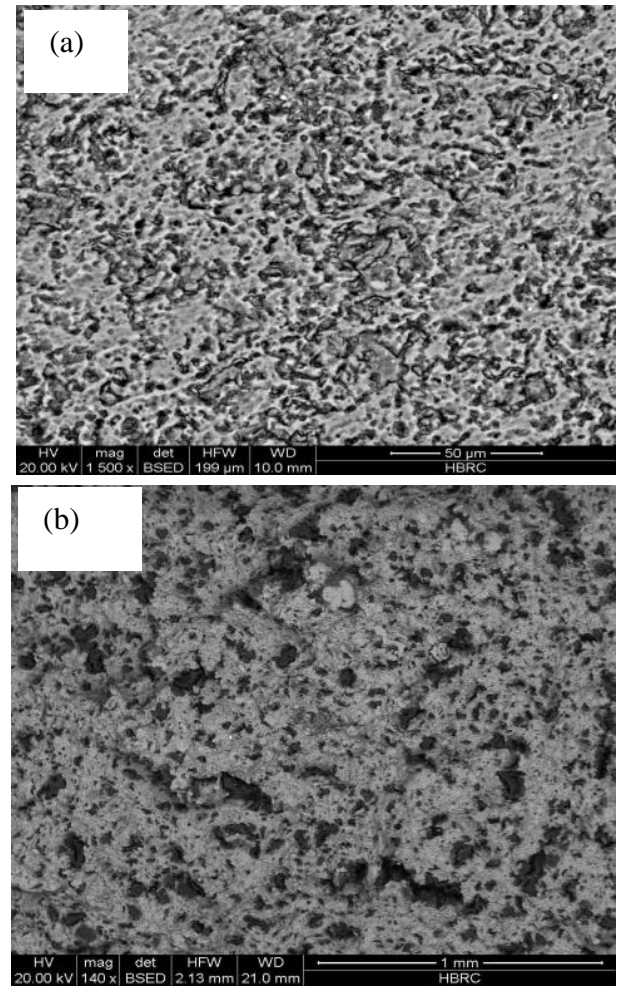

Fig. 5 SEM micrograph of composite after extrusion (a) 2.0 wt.\% CNPLs; (b) 4.0 wt.\% CNPLs.

Figure 6 shows the variation of relative density of composites after extrusion as function of graphite nanoplatelets. High fraction of graphite nanoplatelets gives lower relative density due to lower density of graphite nanoplatelets relative to aluminum. The relative density is shown to be decreased from $97 \%$ to $90.7 \%$ by increasing weight fraction of graphite nanoplatelets from $0 \%$ to $5 \%$.

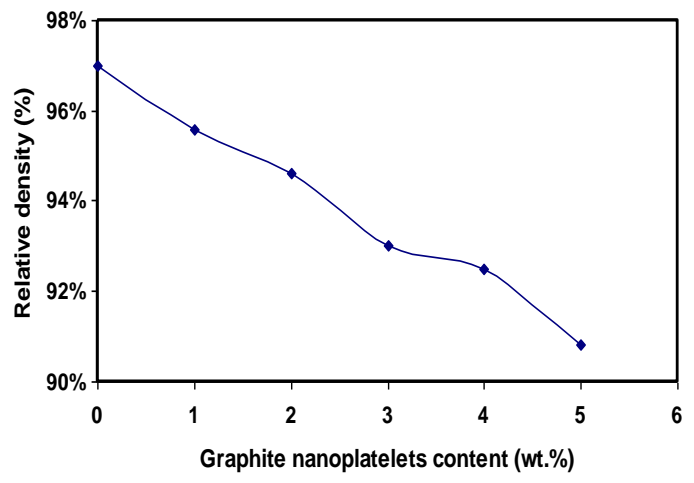

Fig. 6 Effects of Graphite nanoplatelets content on relative density of CNPLs/Al composites.

The effect of the graphite nanoplatelets content on the hardness is shown in Fig. 7. It is evident that with a small amount of graphite nanoplatelets addition, the hardness of the composites increases with increasing graphite nanoplatelets content, while large amount of graphite nanoplatelets reduce the hardness 
of the composites. This may be due to the fact that a small amount of graphite nanoplatelets addition could fill up the microvoids resulting in an increase of the hardness of $\mathrm{CNF}-\mathrm{Al}$ composites. However, a large amount of graphite nanoplatelets are prone to tangle together in blended powders of $\mathrm{Al}$ powders and graphite nanoplatelets. Graphite nanoplatelets conglomeration not only impedes the densification of the CNPLs/Al composites, but also becomes as a defect source. Hence, the hardness of the composites decreases.

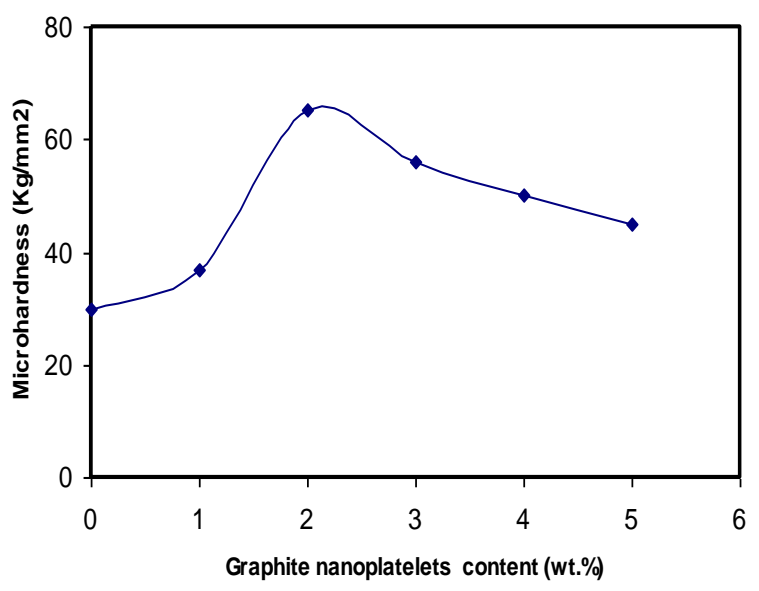

Fig. 7 Effects of Graphite nanoplatelets content on microhardness of CNPLs/Al composites

Figure 8 shows the tensile stress $(\sigma)$ versus strain (ع) curves of the aluminum graphite composites samples tested in the extrusion direction with different CNPLs wt\% graphite contents. From this figure it is evident the increment of the strenght upholding the ductility up to 2 wt.\% CNPLs then decreases for more.

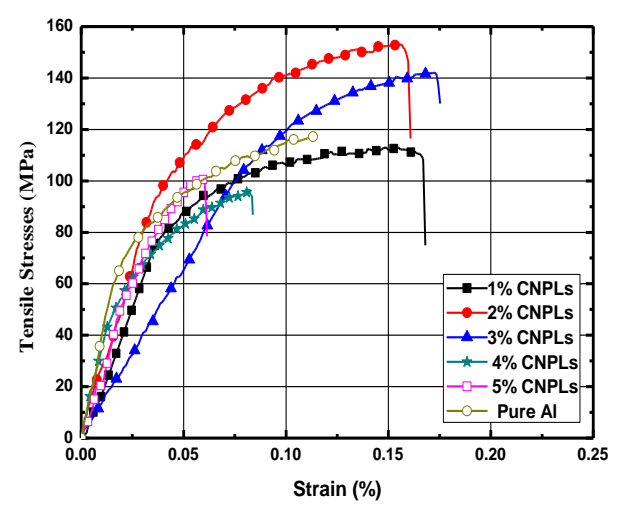

Fig. 8 Tensile stress vs. strain for all samples

Figure 9 presents the proof stress (yield stress $(\sigma y))$ and the ultimate stress $(\sigma \mathrm{u})$ values found in the tensile tests. It can be seen that graphite nanoplatelets content affects significantly $\sigma \mathrm{y}$ and $\sigma \mathrm{u}$ of composites. The $\sigma \mathrm{y}$ and $\sigma \mathrm{u}$ values firstly increase with increasing graphite nanoplatelets content up to
2.0 wt.\%, but decrease obviously with increasing graphite nanoplatelets content.

$\sigma y$ and $\sigma u$ values reach the maximum values (103 $\mathrm{MPa}$ and 154.6 MPa), respectively, when graphite nanoplatelets content is $2.0 \mathrm{wt} . \%$. This phenomenon may be due to the uniform distribution of very small amount of graphite nanoplatelets in the composites leading to dispersion strengthening, and filling up the voids. Meanwhile, the dispersed graphite nanoplatelets restrain the growth of $\mathrm{Al}$ grains during fabrication of the composites bringing on grain refinement strengthening. Therefore, the mechanical properties of the CNPLs/Al composites increase with increasing graphite nanoplatelets content up to 2 wt.\%. However, more quantity of graphite nanoplatelets impede the densification process resulting in a decrease of the relative density of composites, moreover the bonding between graphite nanoplatelets in the conglomeration is very weak, leading to the deterioration in mechanical properties.

It is also very interesting to note that the elongation of composites keeps almost invariable with graphite content up to 2 wt.\% CNPLs. This may be owing to the fact that graphite nanoplatelets can increase the toughness of the composites by absorbing energy because of their highly flexible elastic behavior during loading [9, 10], which is markedly different from the traditional fibers or whiskers. It is generally accepted that the mechanical properties of the composites are dominated not only by the reinforcement and the matrix but also by the interfacial bonding status between them [14].

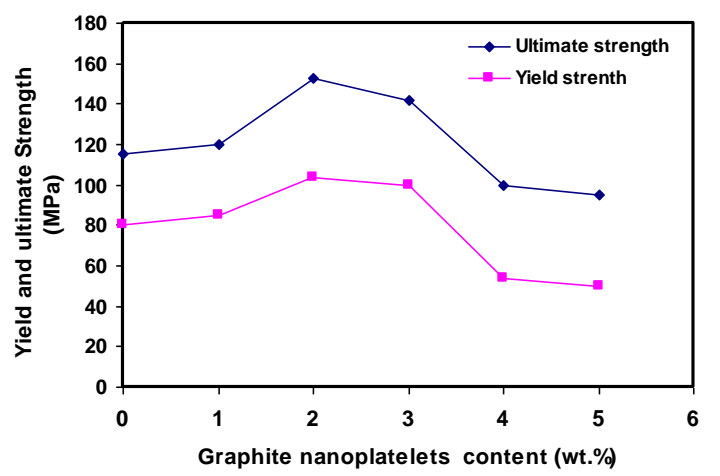

Fig. 9 Effect of Graphite nanoplatelets content on $\sigma y$ and $\sigma \mathrm{u}$

\subsection{The morphology of surface fracture in tensile testing}

It is necessary to study the materials fracture behavior to understand the load transfer between the matrix and reinforcement. Improvements in the strength of graphite nanoplatelets / Al matrix composites are largely attributable to sufficient load transfer from the matrix to graphite nanoplatelets through the interface. To ensure a good load transfer, the composite must maintain a medium strong interfacial bonding. 
It is seen from Fig. 10(a) that the graphite nanoplatelets are uniformly distributed in the matrix; meanwhile, some graphite nanoplatelets are pulledout on the tensile fracture surfaces of composite specimens reinforced with 2.0 wt.\% graphite nanoplatelets.

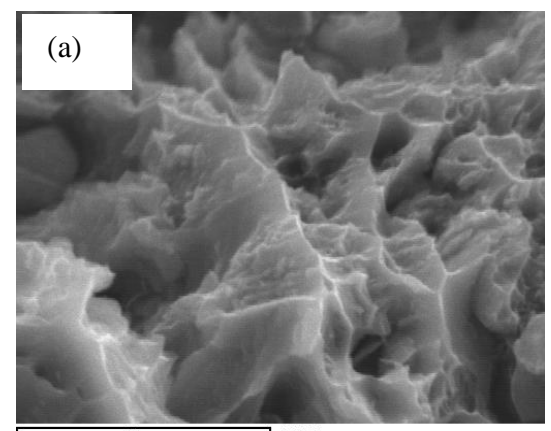

CNPLs

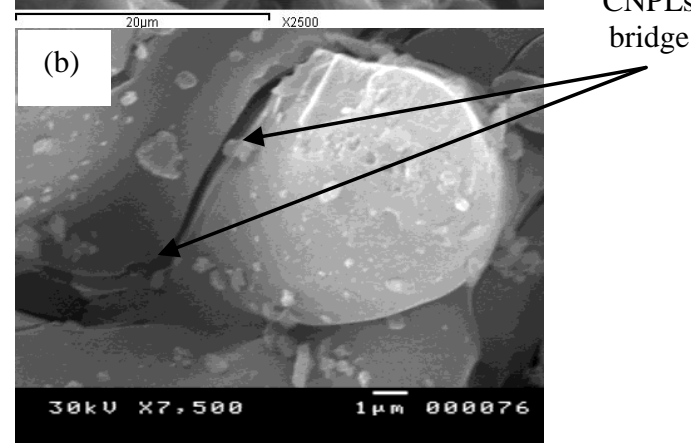

Fig. 10 SEM micrographs of the composites fracture surfaces with $(\mathrm{a}, \mathrm{b}) 2.0 \mathrm{wt} . \%$ CNPLs

The pits on the fracture surface indicate that the length of pulled-out graphite nanoplatelets are very short, suggesting a strong interfacial bonding between graphite nanoplatelets and the Al matrix. Which result in the high mechanical properties obtained in 2.0 wt.\% graphite nanoplatelets/Al composite. Figure 10 (b) shows that the graphite nanoplatelets is bonded to the $\mathrm{Al}$ matrix in "bridging" manner, increasing the CNPLs/Al interface strength and the fracture energy of the composites [15].

However, when 4.0 wt.\% graphite nanoplatelets are added naked graphite nanoplatelets stand on the fracture surface as shown in Fig. 11(a). The higher content of graphite nanoplatelets above $2.0 \mathrm{wt} \%$ indicates that the interface bond between CNPLs and Al matrix is weakly resulting in unsufficient load transfer from the matrix to graphite nanoplatelets through the interface. Moreover, there are some microvoids in the CNPLs composite containing more than 2.0 wt.\% CNPLs as showed in Fig. 11(b), which leads to earlier fracture of the composite under tensile stresses.

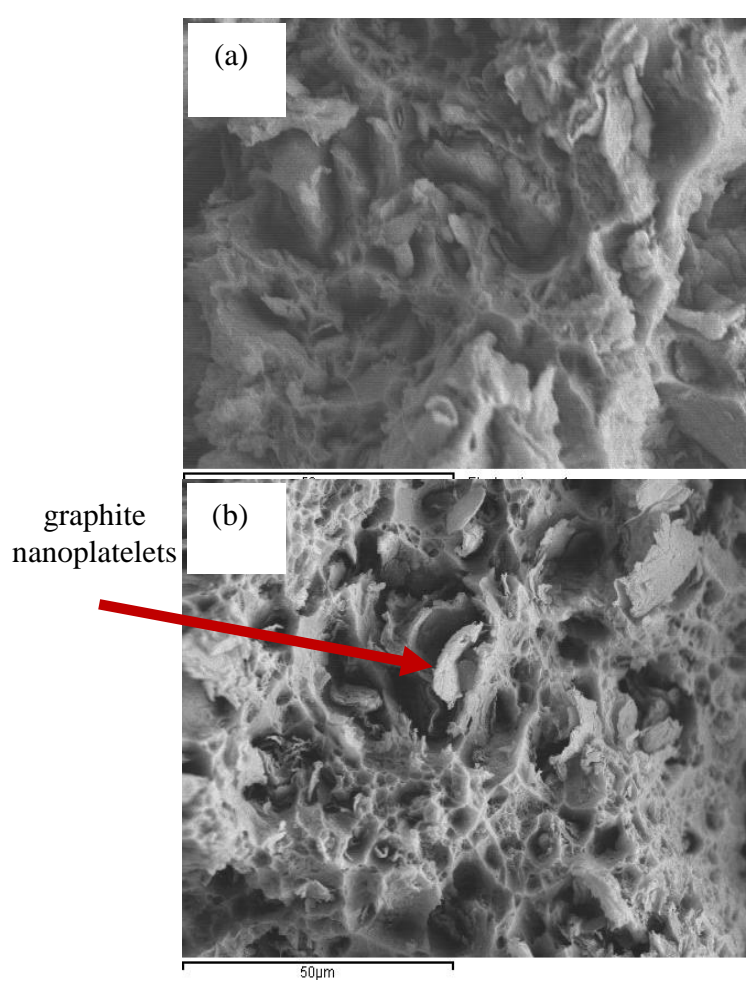

Fig. 11 SEM micrographs of the composites fracture surfaces with $(\mathrm{a}, \mathrm{b}) 4.0 \mathrm{wt} \%$ CNPLs

These microvoids are responsible for the deterioration of the mechanical properties of the composites. In adddition, the interfacial bonding status between the reinforcement particles (CNPLs) and $\mathrm{Al}$ matrix is also another important factor which affects the mechanical properties of composites.

\section{CONCLUSIONS}

- Natural flake graphite with an average size of $500 \mu \mathrm{m}$ in thickness was used for preparing the graphite nanoplatelets by using chemical method (intercalation by sulfuric and nitric acid, exfoliation by thermal shock and then sonocation). The prepared graphite nanoplatlets exhibited an average size of $30-60 \mathrm{~nm}$ in thicknesses.

- The aluminum matrix composites reinforced with CNPLs up to 5 wt.\% were fabricated by cold pressing and then hot extrusion techniques.

- CNPLs have been distributed homogenously on the surfaces of the Al powders by using DMF solution with mechanical stirring.

- The hardness, yield and tensile strengths increase with increasing CNPLs content up to $2.0 \mathrm{wt} \%$, beyond which they decrease. The composite with 2.0 wt.\% CNPLs content exhibits the highest hardness, yield and tensile strengths, which they increased by $108 \%, 29 \%$ and $32 \%$ respectively compared with the Al matrix material fabricated under the same condition. 
- The fracture behavior of CNPLs/Al composites mainly includes "bridging" and "pulling out" of CNPLs on fracture surfaces when CNPLs content is below 2.0 wt.\%, but "interface debonding" occurs when CNPLs is equal 4.0 wt. $\%$.

\section{ACKNOWLEDGMENT}

The authors would like to thank Dr. Adel Fathy and Eng. Mohamed Eltaher, Mechanical Design and Production Engineering Dept., Faculty of Engineering, Zagazig University, for their helpful assistance.

\section{REFERENCES}

[1] S.J. Harris, "Cast metal matrix composites", Mater. Sci. Tech. 4 (1988) 231-239.

[2] P.K. Rohatgi, R. Asthana, S. Das, "solidification, structures, and properties of cast metal-ceramic particle composites", Int. Met. Rev. 31 (3) (1986) 115.

[3] S. Iijima, "Helical microtubules of graphitic carbon", Nature 354 (1991) 56.

[4] KS Novoselov, AK Geim, SV Morozov, D Jiang, Y Zhang, SV Dubonos, "Electric field effect in atomically thin carbon films". Science 2004;306:666-9.

[5] KS Novoselov, AK Geim, SV Morozov, D Jiang, MI Katsnelson, IV Grigorieva, "Twodimensional gas of massless Dirac fermions in grapheme". Nature 2005;438:197-200.

[6] G. Chen, D. Wu, W. Weng, C. Wu, "Exfoliation of graphite flake and its nanocomposites", Letters to the Editor / Carbon 41 (2003) 579 625

[7] W. Li, C. Han, W. Liu, M. Zhang, K. Tao, "Expanded graphite applied in the catalytic process as a catalyst support", Catalysis Today 125 (2007), 278-281.

[8] T. Noguchi, A. Magraio, S. Fukazawa, S. Shimizu, J. Beppu, M. Seki, "Carbon Nanotube/Aluminium Composites with Uniform Dispersion", Mater. Trans. 45 (2004) 602-604.

[9] S.I. Cha, K.T. Kim, S.N. Arshad, C.B. Mo, S.H. Hong, "Extraordinary Strengthening Effect of Carbon Nanotubes in Metal-Matrix Nanocomposites Processed by Molecular-Level Mixing", Adv. Mater. 17 (2005) 1377-1381.

[10] X. Hu, T. Wang, X. Qu, S. Dong, "Noncovalent Functionalization of Multiwalled Carbon Nanotubes: Application in Hybrid Nanostructures" , J. Phys. Chem. B 110 (2) (2006) 853-857.

[11] GH Chen, DJ Wu, WG Weng, CL Wu. "Exfoliation of graphite flake and its nanocomposites". Carbon 2003;41:619-21.

[12] G.-D. Zhan, J.D. Kuntz, J. Wan, A.K. Mukherhee, "Single-wall carbon nanotubes as attractive toughening agents in alumina-based nanocomposites", Nat. Mater. 2 (2003) 38-42.

[13] M.I. Flores, I. Estrada, J. Gonzalez, M. Miki, R. Mart, "Aluminum-graphite composite produced by mechanical milling and hot extrusion", Journal of Alloys and Compounds 434-435 (2007) 518-521

[14] L.M. Tham, M. Gupta, L. Chen, "Effect of limited matrix-reinforcement interfacial reaction on enhancing the mechanical properties of aluminium-silicon carbide composites", Acta Mater. 49 (2001) 32433253.

[15] P.M. Ajayan, L.S. Schadler, C. Giannaris, A. Rubio, "Single-Walled Carbon Nanotube Polymer Composites: Strength and Weakness", Adv. Mater. 12 (10) (2000) 750-753. 